\title{
Anticoagulation and population risk of stroke and death in incident atrial fibrillation: a population-based cohort study
}

\author{
Amy Y.X. Yu MD, Shaun Malo MSc, Stephen Wilton MD, Ratika Parkash MD MS, \\ Lawrence W. Svenson BSc DipPsych, Michael D. Hill MD MSc
}

Abstract

Background: Atrial fibrillation increases the risk of stroke and death. Anticoagulation therapy is an effective treatment for stroke prevention, but remains underused in the community. We sought to determine the effectiveness and safety of anticoagulation therapy in an inception cohort with new-onset atrial fibrillation in the province of Alberta, Canada.

Methods: We conducted a population-based cohort study of atrial fibrillation using an administrative database from Alberta's publicly funded and universally available health care system. All new-onset atrial fibrillation patients from Jan. 1, 2009, to Dec. 31, 2010, were included in the cohort and followed through Dec. 31, 2013. We assessed anticoagulation status as a predictor of stroke and death using time-to-event analysis and adjusted for sex and CHADS2 (congestive heart failure, hypertension, age $\geq 75 \mathrm{yr}$, diabetes mellitus and prior stroke or transient ischemic attack) score using Cox proportional hazards modelling.

Results: We identified 10745 patients, 7358 (68.5\%) of whom received anticoagulation therapy, principally with warfarin $(n=6997$, 95.1\%). Anticoagulation therapy was associated with significantly decreased risk of ischemic stroke (hazard ratio [HR] $0.69,95 \%$ confi-

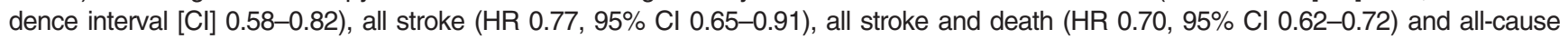
mortality (HR 0.67, 95\% Cl 0.62-0.72), despite an association with increased risk of hemorrhagic stroke (HR 1.92, 95\% Cl 1.17-3.16). There was a neutral association with subdural (HR 1.01, 95\% Cl 0.53-1.93) and gastrointestinal ( $\mathrm{HR} 0.96,95 \% \mathrm{Cl} \mathrm{0.70-1.31)} \mathrm{hemorrhage.}$

Interpretation: Anticoagulation therapy is effective and safe for stroke prevention and decreases mortality in patients with incident atrial fibrillation. These population data support an aggressive approach to screening for atrial fibrillation and treatment with anticoagulant medicines to prevent stroke and death.

trial fibrillation is the most common cardiac arrhythmia, affecting $1 \%-2 \%$ of the Western population and increasing in prevalence with advancing age. ${ }^{1,2}$ Compared with patients without atrial fibrillation, the arrhythmia is associated with 2-fold greater mortality and 5-fold greater risk of ischemic stroke. ${ }^{3,4}$ These cardioembolic ischemic strokes are associated with higher rates of disability compared with ischemic stroke from other causes. ${ }^{5}$ Warfarin is an inexpensive and effective stroke prevention strategy, but it has a narrow therapeutic index, and close monitoring of the international normalized ratio is required. The mean time in the therapeutic range lies between $60 \%$ and $70 \%$ in the setting of clinical trials, but it may be $50 \%$ or lower in routine clinical practice. ${ }^{6-8}$ Anticoagulation therapy for atrial fibrillation does not show a mortality benefit in single clinical trials, but a mortality reduction has been reported with meta-analyses of multiple studies. ${ }^{9-11}$ Despite these benefits, orally administered anticoagulation therapy is underused on a population basis. ${ }^{12-14}$

Anticoagulation impairs clotting. Therefore, it can uncover or worsen any spontaneous or induced hemorrhage from another cause. Nuisance bleeding, such as skin bruising, is common. Gastrointestinal hemorrhage is the most common serious form of hemorrhage, but intracranial hemorrhage is the complication most feared by patients and physicians alike.

To assess the population benefit of oral anticoagulation therapy, we used contemporary and complete population data to investigate population rates of stroke, death and the most common complications of the therapy - gastrointestinal and subdural hemorrhage - among patients with a new diagnosis of atrial fibrillation in the province of Alberta, Canada.

\section{Methods}

\section{Study design and population}

We conducted a population-based retrospective cohort study of incident atrial fibrillation using administrative data

Competing interests: See the end of the article.

This article has been peer reviewed.

Correspondence to: Michael D. Hill, michael.hill@ucalgary.ca CMAJ Open 2016. DOI:10.9778/cmajo.20150096 
from the province of Alberta. All Alberta residents (population 4025078 in $2013^{15}$ ) are eligible for a publicly funded and universally available health care system. The Alberta Health Care Insurance Plan provides medical coverage to most Alberta residents (99\%). The only exceptions are members of the military and Royal Canadian Mounted Police, federal inmates and people who opt out of the plan. Each resident covered by the plan is assigned a personal health number that acts as a unique lifetime identifier. A linked Pharmaceutical Information Network records all prescription drug use from outpatient pharmacies. Data were extracted from the Alberta Health hospital inpatient, ambulatory/emergency department encounters, physician claims and Pharmaceutical Information Network databases. Canadian administrative data have been previously shown to be valid and highly accurate. ${ }^{16,17}$

We defined an inception cohort of patients with new-onset atrial fibrillation from Jan. 1, 2009, to Dec. 31, 2010, and followed them through Dec. 31, 2013. Atrial fibrillation was identified using International Classification of Diseases 9th revision (clinical modification) (ICD-9-CM) code 427.3x or International Classification of Diseases and Related Health Problems, 10th revision, Canadian edition (ICD-10-CA) code I48. $x$ in any diagnosis field in any of the Alberta Health hospital inpatient, ambulatory/emergency department encounters or physician claims databases. Atrial fibrillation was defined as new-onset if no previous diagnosis of atrial fibrillation had been made in Alberta from the date the patient obtained an Alberta Health Care Insurance Plan number or Apr. 1, 1994. Rarely, a new migrant to the province during the study period may have been included in the cohort as a patient with newonset atrial fibrillation even if the diagnosis was made elsewhere. Two diagnoses for atrial fibrillation were required at separate health care encounters more than 30 days apart within the first year of diagnosis to meet the case definition and minimize misclassification of transient single episodes of atrial fibrillation or flutter. Patients with valvular heart disease were excluded if the following codes appeared in any of the databases preceding the incidence date: mitral or aortic disease (ICD-9-CM 394-396, 424.0, 424.1 or ICD-10 I05, I06, I34, I35, I08.0, I08.1, I08.2, I08.3) or valve surgery (ICD9-CM 35.0x, 35.2x, 35.96, 35.97, 35.99 and ICD-10-CA 1. HT.89, 1.HV.80, 1.HU.80, 1.HT.80, 1.HS.80, 1.HV.90, 1. HU.90, 1.HT.90, 1.HS.90). ${ }^{17,18}$

The composite of all stroke and all-cause mortality was the primary outcome. Secondary outcomes were individual components of the composite outcome and rates of gastrointestinal and subdural hemorrhage. Stroke was divided into ischemic and hemorrhagic types. Ischemic stroke was defined as any hospital admission with ICD-9-CM codes 362.3, 433.x1, 434. x1, 436 or ICD-10-CA codes H34.1, H34.2, I63.x, I64.x. Similarly, hemorrhagic stroke was defined using ICD-9-CM codes 430.x, 431.x or ICD-10-CA codes I60.x, I61.x. ${ }^{17} \mathrm{We}$ examined subdural hemorrhage (ICD-9-CM 432.x or ICD-10-CA I62.x as the primary diagnosis) separately because subdural hemorrhage is not a stroke; it is a specific bleeding complication principally associated with trauma and made more serious with anticoagulation. We also examined rates of gastrointestinal bleeding (ICD-10-CA codes K25, K26, K27, K28, K29 as the primary diagnosis). ${ }^{19}$

Patients were divided into primary and secondary prevention cohorts. The primary prevention cohort consisted of patients who met the case definition for atrial fibrillation without a previous cerebrovascular event in Alberta from the date the patient obtained an Alberta Health Care Insurance Plan number or Apr. 1, 1994. In contrast, the secondary prevention cohort consisted of patients with a previous diagnosis of stroke (ischemic or hemorrhage) or transient ischemic attack, including patients with concurrent diagnoses of atrial fibrillation and stroke. Rarely, patients who migrated to the province with a history of transient ischemic attack or stroke were assigned to the primary prevention cohort because prior stroke status could not be determined. A small number of patients in the secondary prevention cohort had CHADS2 (congestive heart failure, hypertension, age $\geq 75 \mathrm{yr}$, diabetes mellitus and prior stroke or transient ischemic attack) scores of 0 or 1 because they had suffered a previous hemorrhagic event.

The Pharmaceutical Information Network was linked to the Alberta Health hospital inpatient, ambulatory/emergency department encounters and physician claims databases by using unique health care numbers to assess prescriptions for anticoagulant medications. The Pharmaceutical Information Network contains records for all pharmaceuticals dispensed by community pharmacies in Alberta. Each record contains the unique health care number, the drug identification number of the pharmaceutical dispensed and the Anatomic Therapeutic Chemical Classification System code that classifies drugs based on organ or body system. We considered oral anticoagulants only, including warfarin and the direct oral anticoagulant medications (Anatomic Therapeutic Chemical codes B01AA, B01AF, B01AX06 and B01AE07). Patients were considered to have undergone anticoagulation therapy if they had been prescribed oral anticoagulants within the 6 months before an outcome or the last date of follow-up, whichever was first, and if they had at least 1 renewal of the prescription. Because acetylsalicylic acid (ASA) is available over the counter, it could not be reliably assessed. In addition, the Pharmaceutical Information Network only captures outpatient prescriptions. Therefore, anticoagulants prescribed for patients admitted to an Alberta hospital or long-term care institution may not be captured. We assessed anticoagulation status as a predictor of stroke and death.

\section{Statistical methods}

Patient characteristics were described using standard descriptive statistics. We used time-to-event analysis to estimate the risk of each outcome. Patient data were censored if they moved away from the province before the end of the study. We adjusted for sex and CHADS2 score, defined by administrative data, using Cox proportional hazards modelling. ${ }^{16,20,21}$ A sensitivity analysis was conducted to risk-adjust for baseline comorbidities by applying the Elixhauser comorbidity index to the subpopulation of patients who received a diagnosis of atrial fibrillation while admitted to hospital. ${ }^{22}$ 


\section{Results}

From a population of 4 million Alberta residents, a total of 10745 patients with newly diagnosed atrial fibrillation were identified, of whom 1203 (11.2\%) had a previous cerebrovascular event (Table 1). Of the identified patients, 7358 (68.5\%) were prescribed anticoagulation therapy at least once, 6997 (95.1\%) of whom received warfarin and 1430 (19.4\%) of whom received a direct oral anticoagulant. (The sum of warfarin and direct oral anticoagulant use was more than $100 \%$, because a number of patients were prescribed both medications, serially, during the study period.) A total of 554 strokes occurred during the follow-up period. Among the 747 patients with new-onset atrial fibrillation and a previous ischemic stroke, 214 (28.6\%) were given a first-ever diagnosis of atrial fibrillation during the same hospital admission as the stroke; data from 172 (1.6\%) patients were censored because the patients moved away from the province before the end of the study.

Anticoagulation therapy was consistently associated with decreased risk of stroke and death in all cohorts (Table 2 and Figure 1). There was no evidence that CHADS2 score modified the protective effect of anticoagulation status ( $p$ for interaction $=0.74$ ). Anticoagulation prescription was also associated with reduction in the secondary outcomes of ischemic stroke, all stroke and death. Of the 500 acute ischemic stroke events, $25(5.0 \%)$ were in residents of long-term care institu- tions. Similarly, 537 (17.3\%) of the 3097 composite outcomes of all stroke or all-cause mortality occurred in residents of long-term care facilities. In the subgroup of patients with atrial fibrillation admitted to hospital $(n=3598)$, the beneficial association with all stroke and death remained true after adjustment for the Elixhauser comorbidity index (Table 3).

Anticoagulation was associated with increased intracranial hemorrhage in the total population but not in the individual primary or secondary prevention cohorts (Table 2). There was a neutral association with the risks of gastrointestinal hemorrhage $(n=158)$ (hazard ratio [HR] 0.96, 95\% confidence interval [CI] 0.70-1.31) and subdural hemorrhage $(n=37)$ (HR 1.01, 95\% CI 0.53-1.93).

\section{Interpretation}

\section{Main findings}

In a large population with universal health care coverage and high rates of anticoagulation therapy for atrial fibrillation, orally administered anticoagulation was associated with reduced risk of stroke and death. This association was true with and without a history of previous stroke. ${ }^{11,23}$ Stratification by CHADS2 scores did not change this result. In $28.6 \%$ of patients with stroke and new-onset atrial fibrillation, stroke was the presenting complication of atrial fibrillation. Anticoagulation was associated with an increased risk of intracra-

Table 1: Baseline characteristics

\begin{tabular}{|c|c|c|c|c|c|c|}
\hline \multirow[b]{2}{*}{ Characteristic } & \multicolumn{2}{|c|}{$\begin{array}{l}\text { All patients } \\
(n=10745)\end{array}$} & \multicolumn{2}{|c|}{$\begin{array}{l}\text { Primary prevention } \\
\qquad(n=9568)\end{array}$} & \multicolumn{2}{|c|}{$\begin{array}{l}\text { Secondary prevention } \\
\qquad(n=1177)\end{array}$} \\
\hline & $\begin{array}{c}\text { Underwent } \\
\text { anticoagulation } \\
\text { therapy }\end{array}$ & $\begin{array}{l}\text { Did not undergo } \\
\text { therapy }\end{array}$ & $\begin{array}{c}\text { Underwent } \\
\text { anticoagulation } \\
\text { therapy }\end{array}$ & $\begin{array}{l}\text { Did not undergo } \\
\text { therapy }\end{array}$ & $\begin{array}{l}\text { Underwent } \\
\text { anticoagulation } \\
\text { therapy }\end{array}$ & $\begin{array}{l}\text { Did not undergo } \\
\text { therapy }\end{array}$ \\
\hline Participants, no. (\%) & $7358(68.5)$ & 3387 (31.5) & $6497(67.9)$ & $3071(32.1)$ & 861 (73.2) & $316(26.8)$ \\
\hline Age, yr, median (IQR) & $74(17)$ & $70(27)$ & $74(18)$ & $68(27)$ & $78(14)$ & $83(15)$ \\
\hline Male sex, no. (\%) & $4149(56.4)$ & $1785(52.7)$ & $3710(57.1)$ & $1638(53.3)$ & $439(51.0)$ & $147(46.5)$ \\
\hline Hypertension, no. (\%) & $3461(47.0)$ & $1333(39.4)$ & $2991(46.0)$ & $1168(38.0)$ & $470(54.6)$ & 165 (52.2) \\
\hline Diabetes, no. (\%) & $1833(24.9)$ & $645(19.0)$ & $1585(24.4)$ & $546(17.8)$ & $248(28.8)$ & 99 (31.3) \\
\hline $\begin{array}{l}\text { Ischemic stroke and/or transient } \\
\text { ischemic attack, no. (\%) }\end{array}$ & $843(11.5)$ & $301(8.9)$ & 0 & 0 & $843(97.9)$ & 301 (95.3) \\
\hline Ischemic stroke, no. (\%) & $561(7.6)$ & $186(5.5)$ & 0 & 0 & $561(65.2)$ & $186(58.9)$ \\
\hline Hemorrhagic stroke, no. (\%) & $34(0.5)$ & $25(0.7)$ & 0 & 0 & $34(3.9)$ & $25(7.9)$ \\
\hline \multicolumn{7}{|l|}{ CHADS 2 score, no. (\%) } \\
\hline 0 & $1553(21.1)$ & $1140(33.7)$ & $1551(23.9)$ & $1138(37.1)$ & $2(0.2)$ & $2(0.6)$ \\
\hline 1 & 2550 (34.7) & 1089 (32.2) & 2544 (39.2) & $1082(35.2)$ & $6(0.7)$ & $7(2.2)$ \\
\hline 2 & 2029 (27.6) & 709 (20.9) & $1935(29.8)$ & 672 (21.9) & 94 (10.9) & 37 (11.7) \\
\hline 3 & 795 (10.8) & 266 (7.9) & $445(6.8)$ & $168(5.5)$ & $350(40.7)$ & $98(31.0)$ \\
\hline 4 & 354 (4.8) & $153(4.5)$ & $22(0.3)$ & $11(0.4)$ & $332(38.6)$ & $142(44.9)$ \\
\hline 5 & $76(1.0)$ & $27(0.8)$ & 0 & 0 & 76 (8.8) & 27 (8.5) \\
\hline 6 & $1(0)$ & $3(0)$ & 0 & 0 & $1(0)$ & $3(0.9)$ \\
\hline
\end{tabular}




\section{OPEN}

Research

nial hemorrhage, but the effect size was variable, the absolute rate was low, and the association did not negate the beneficial association with all strokes (hemorrhagic and ischemic strokes combined) or all stroke and death. Gastrointestinal hemorrhage and subdural hemorrhage were not associated with anticoagulation status, but the number of events was very low.

The all-cause mortality reduction seen in our study may be due to the high rates of anticoagulant prescription $(68.5 \%)$, comparable to randomized controlled trials. In contrast to Alberta data from more than a decade earlier, the rates of anticoagulation show a substantial $20 \%$ absolute increase. ${ }^{14}$ Although it is not known what an expected ceiling rate of appropriate anticoagulation might be, a recent population study from the United Kingdom showed that, despite a rising trend in anticoagulation rates in the last decade, only $58 \%$ of men and $52 \%$ of women with atrial fibrillation received anticoagulation orally in $2012 .^{12}$

Stroke was the initial presenting symptom of atrial fibrillation in more than 1 in 4 patients with a history of acute ischemic stroke. Similarly, a recent nation-wide Swedish study shows that one third of patients with acute ischemic stroke have atrial fibrillation. ${ }^{24}$ Because anticoagulation is such an effective therapy, the question remains as to whether more aggressive strategies for screening and diagnosis of atrial fibrillation in the community, including prolonged electrocardiography or the use of implantable recording devices, would lead to better prevention of stroke and cardiovascular events. ${ }^{13,25,26}$ Whether or not the temporal relationship between new atrial fibrillation and stroke can be definitively interpreted as a causal relationship remains controversial. ${ }^{27,28}$ In a trial involving patients with implanted pacemakers, some patients with atrial fibrillation had the arrhythmia recorded after they had suffered a stroke. ${ }^{25} \mathrm{Nev}$ ertheless, extended cardiac monitoring detecting new paroxysmal atrial fibrillation in the acute period after ischemic stroke is highly predictive of chronic paroxysmal atrial fibrillation. ${ }^{29}$ Current guidelines suggest that these patients should receive lifelong anticoagulation therapy. ${ }^{30}$

In our study, hemorrhagic complications were low. This is consistent with the pathophysiological knowledge that anticoagulant medications impair thrombosis, but are not the direct cause of hemorrhage. For example, spontaneous intracranial hemorrhage, commonly due to chronic hypertension or underlying amyloid angiopathy, is more severe among patients who underwent anticoagulation therapy. ${ }^{31}$ A recent longitudinal population-based study showed a decreasing trend in incidence and mortality related to warfarin-related intracerebral hemorrhages despite a 4-fold increase in warfarin use. ${ }^{32}$ The appropriate use of anticoagulation among patients with nonvalvular atrial fibrillation may also be a marker of better overall

Table 2: Outcomes according to anticoagulation status

\begin{tabular}{|c|c|c|c|c|c|}
\hline \multirow[b]{2}{*}{ Subgroup; outcome } & \multicolumn{2}{|c|}{ Underwent anticoagulation therapy } & \multicolumn{2}{|c|}{$\begin{array}{c}\text { Did not undergo anticoagulation } \\
\text { therapy }\end{array}$} & \multirow[b]{2}{*}{$\mathrm{HR}(95 \% \mathrm{Cl}) \dagger$} \\
\hline & Events, no. & Event rate $(95 \% \mathrm{Cl})^{*}$ & Events, no. & Event rate $(95 \% \mathrm{Cl})^{*}$ & \\
\hline \multicolumn{6}{|c|}{ All patients $(n=10745)$} \\
\hline All stroke and death & 1492 & $73.7(70.0-77.7)$ & 1605 & $112.6(107.0-118.3)$ & $0.70(0.62-0.72)$ \\
\hline Ischemic stroke & 237 & $11.8(10.3-13.5)$ & 263 & $18.0(15.8-20.4)$ & $0.69(0.58-0.82)$ \\
\hline Hemorrhagic stroke & 56 & $2.8(2.1-3.7)$ & 22 & $1.5(1.0-2.4)$ & $1.92(1.17-3.16)$ \\
\hline All stroke & 279 & $14.0(12.3-15.8)$ & 276 & $19.0(16.8-21.5)$ & $0.77(0.65-0.91)$ \\
\hline Death & 1338 & $63.8(60.4-67.4)$ & 1478 & $102.0(96.8-107.4)$ & $0.67(0.62-0.72)$ \\
\hline \multicolumn{6}{|c|}{ Primary prevention cohort $(n=9568)$} \\
\hline All stroke and death & 1265 & $70.6(66.7-74.7)$ & 1361 & $103.7(98.2-109.5)$ & $0.70(0.65-0.76)$ \\
\hline Ischemic stroke & 171 & $9.7(8.3-11.4)$ & 216 & $15.9(13.8-18.3)$ & $0.62(0.51-0.76)$ \\
\hline Hemorrhagic stroke & 41 & $2.3(1.7-3.3)$ & 19 & $1.4(0.9-2.3)$ & $1.71(0.98-2.96)$ \\
\hline All stroke & 203 & $11.7(10.1-13.5)$ & 227 & $16.9(14.7-19.3)$ & $0.71(0.58-0.85)$ \\
\hline Death & 1149 & $61.9(58.3-65.7)$ & 1248 & $94.2(89.0-99.7)$ & $0.68(0.63-0.74)$ \\
\hline \multicolumn{6}{|c|}{ Secondary prevention $(n=1177)$} \\
\hline All stroke and death & 227 & $103.9(90.7-120.1)$ & 244 & $190.7(167.2-217.8)$ & $0.55(0.46-0.66)$ \\
\hline Ischemic stroke & 66 & $31.0(23.9-41.5)$ & 47 & $37.1(27.0-50.9)$ & $0.89(0.61-1.30)$ \\
\hline Hemorrhagic stroke & 15 & $6.4(3.6-13.2)$ & 3 & $2.2(0.42-8.9)$ & $2.96(0.86-10.25)$ \\
\hline All stroke & 76 & $35.5(27.9-46.4)$ & 49 & $38.6(28.4-52.7)$ & $0.97(0.67-1.39)$ \\
\hline Death & 189 & 81.5 (70.3-95.7) & 230 & $168.2(146.9-192.8)$ & $0.49(0.40-0.59)$ \\
\hline
\end{tabular}


care, including treatment of hypertension. Spontaneous hemorrhagic transformation of ischemic stroke is not distinguished from primary intracerebral hemorrhage using administration data. Hemorrhagic transformation is common after cardioembolic ischemic strokes and may be secondarily reduced due to the expected reduction in ischemic stroke. ${ }^{33}$ The perceived risk of serious hemorrhage is a major reason for nonprescription of anticoagulation. ${ }^{12,34}$ Our study supports the use of anticoagulants in this population because the risks of hemorrhage are outweighed by the benefit of decreased ischemic stroke and mortality. Moreover, the risks may be further attenuated by the use of direct oral anticoagulants (e.g., dabigatran, apixaban, rivaroxaban and edoxaban), which are potentially safer and easier to use than warfarin. ${ }^{35}$

\section{Limitations}

Our paper contains limitations, including those inherent to studies using administrative data. The rate of stroke outcomes in our study is similar to the rate seen in the clinical trials. ${ }^{4}$ However, our outcomes were adjusted for CHADS2 score instead of CHA2DS2-VASc (vascular disease, age 65-74 yr, sex category) score, which may be superior in predicting patients at high risk. The reason is vascular disease, including peripheral arterial disease, complex aortic plaque and myocardial infarction, is not well validated in administrative data. ${ }^{36}$ Moreover, misclassification of anticoagulation status could not be completely ruled out because the Alberta Pharmaceutical Information Network data do not include all patients admitted to hospital or a long-term care facility. Nevertheless, the occurrence of events in a long-term care institution was relatively low, meaning that misclassification would have only a marginal effect on the point estimates. In addition, the Pharmaceutical Information Network does not allow us to ascertain whether patients are continuously receiving anticoagulation between the drug dispensation dates. International normalized ratio levels are not linked to the administrative data we used. Most of the patients receiving anticoagulation included in our study were receiving warfarin. The effect of the direct oral anticoagulants may be more substantial in terms of stroke prevention, mortality reduction and reduced bleeding risk on a population level, particularly given the findings from recent clinical trials. ${ }^{6,7,37,38}$ Finally, the Elixhauser comorbidity index has only been validated for inpatients and therefore could not be applied to the entire population, which primarily includes outpatients. ${ }^{22}$ In the subgroup of inpatient atrial fibrillation patients, likely the sickest patients, adjustment for the 30 comorbidities of the Elixhauser index, which includes various conditions such as organ failure, AIDS/HIV, malignant disease and psychiatric disorders, did not significantly change the direction of effect for our primary outcome. Nevertheless, there still remains a possibility that there is confounding by indication: patients with less comorbid illness may be judged to have the highest likelihood of benefit from anticoagulation and are therefore given treatment. Patients

\begin{tabular}{|c|c|}
\hline Outcome & $\mathrm{HR}(95 \% \mathrm{Cl})^{*}$ \\
\hline All stroke and death & $0.61(0.55-0.67)$ \\
\hline Ischemic stroke & $0.84(0.64-1.11)$ \\
\hline Hemorrhagic stroke & $2.18(0.97-4.89)$ \\
\hline All stroke & $0.95(0.73-1.23)$ \\
\hline Death & $0.57(0.52-0.64)$ \\
\hline
\end{tabular}

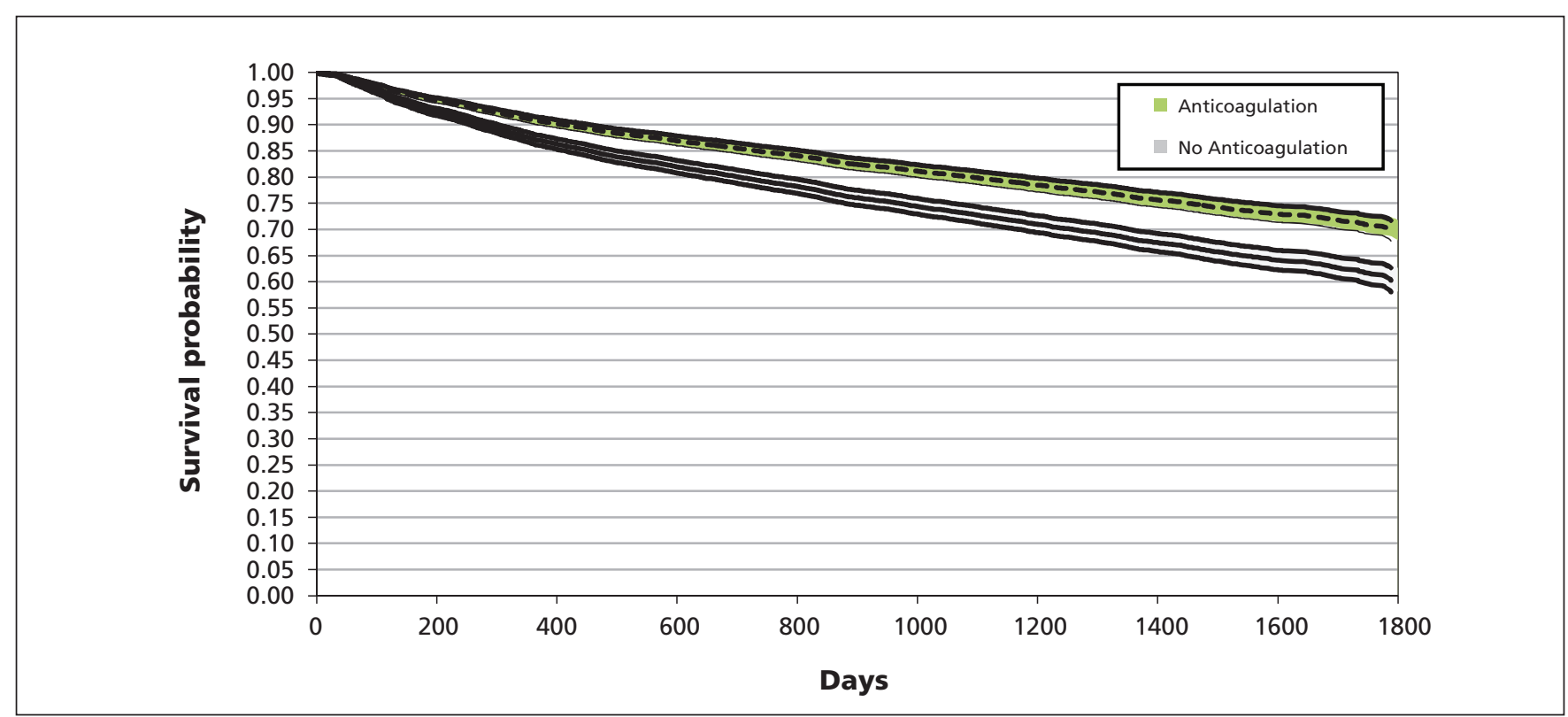

Figure 1: Cox survival curve for all stroke and death, with anticoagulation as a static covariate. 
who have multiple comorbid illnesses are at higher risk of stroke, but they may also be the most likely to experience harm, and therefore are not given anticoagulation. We addressed this issue by defining anticoagulation status in a conservative manner by including all patients who received an oral anticoagulant within 6 months of their outcome event.

\section{Conclusion}

Analyses of large population data sets are adjuncts to randomized controlled trials in which follow-up may not have been long enough or the sample size large enough to detect longer term treatment effects, including mortality benefit. On a population basis, anticoagulation is an effective and safe stroke prevention strategy, associated with decreased mortality among patients with incident atrial fibrillation in real-world practice. The optimal strategy to screen for atrial fibrillation as a primary or secondary stroke prevention strategy needs to be determined.

\section{References}

1. Lip GY, Tse HF. Management of atrial fibrillation. Lancet 2007;370:604-18.

2. Wolf PA, Abbott RD, Kannel WB. Atrial fibrillation as an independent risk factor for stroke: the Framingham Study. Stroke 1991;22:983-8.

3. Benjamin EJ, Wolf PA, D'Agostino RB, et al. Impact of atrial fibrillation on the risk of death: the Framingham Heart Study. Circulation 1998;98:946-52.

4. Hart RG, Halperin JL, Pearce LA, et al. Lessons from the Stroke Prevention in Atrial Fibrillation trials. Ann Intern Med 2003;138:831-8.

5. Andrew N, Kilkenny M, Harris D, et al. Outcomes for people with atrial fibrillation in an Australian national audit of stroke care. Int 7 Stroke 2014;9:270-7.

6. Connolly SJ, Ezekowitz MD, Yusuf S, et al. Dabigatran versus warfarin in patients with atrial fibrillation. N Engl f Med 2009;361:1139-51.

7. Granger CB, Alexander JH, McMurray JJ, et al. Apixaban versus warfarin in patients with atrial fibrillation. N Engl 7 Med 2011;365:981-92.

8. Dlott JS, George RA, Huang X, et al. National assessment of warfarin anticoagulation therapy for stroke prevention in atrial fibrillation. Circulation 2014; 129:1407-14.

9. Stroke Prevention in Atrial Fibrillation Study. Final results. Circulation 1991; 84:527-39.

10. van Walraven C, Hart RG, Singer DE, et al. Oral anticoagulants vs aspirin in nonvalvular atrial fibrillation: an individual patient meta-analysis. $7 A M A$ 2002;288:2441-8

11. Hart RG, Pearce LA, Aguilar MI. Meta-analysis: antithrombotic therapy to prevent stroke in patients who have nonvalvular atrial fibrillation. Ann Intern Med 2007;146:857-67.

12. Scowcroft AC, Cowie MR. Atrial fibrillation: improvement in identification and stroke preventive therapy - data from the UK Clinical Practice Research Datalink, 2000-2012. Int 7 Cardiol 2014;171:169-73.

13. Gladstone DJ, Spring M, Dorian P, et al. Atrial fibrillation in patients with cryptogenic stroke. N Engl 7 Med 2014;370:2467-77.

14. Sandhu RK, Bakal JA, Ezekowitz JA, et al. Risk stratification schemes, anticoagulation use and outcomes: the risk-treatment paradox in patients with newly diagnosed non-valvular atrial fibrillation. Heart 2011;97:2046-50.

15. Alberta Interactive Health Data Application. Government of Alberta. Available: www.ahw.gov.ab.ca/IHDA_Retrieval/redirectToURL.do? cat=5 \&subCat=63 (accessed 2014 Nov. 27).

16. So L, Evans D, Quan H. ICD-10 coding algorithms for defining comorbidities of acute myocardial infarction. BMC Health Serv Res 2006;6:161.

17. Kokotailo RA, Hill MD. Coding of stroke and stroke risk factors using international classification of diseases, revisions 9 and 10. Stroke 2005;36:1776-81.

18. Jensen PN, Johnson K, Floyd J, et al. A systematic review of validated methods for identifying atrial fibrillation using administrative data. Pharmacoepidemiol Drug Saf 2012;21(Suppl 1):141-7.

19. Larsen TB, Rasmussen LH, Skjoth F, et al. Efficacy and safety of dabigatran etexilate and warfarin in "real-world" patients with atrial fibrillation: a prospective nationwide cohort study. 7 Am Coll Cardiol 2013;61:2264-73.

20. Borzecki AM, Wong AT, Hickey EC, et al. Identifying hypertension-related comorbidities from administrative data: What's the optimal approach? $A m \mathcal{F}$ Med Oual 2004;19:201-6.

21. Dublin S, French B, Glazer NL, et al. Risk of new-onset atrial fibrillation in relation to body mass index. Arch Intern Med 2006;166:2322-8.

22. Southern DA, Quan H, Ghali WA. Comparison of the Elixhauser and Charlson/ Deyo methods of comorbidity measurement in administrative data. Med Care 2004;42:355-60.

23. Connolly S, Pogue J, Hart R, et al.; ACTIVE Writing Group of the ACTIVE
Investigators Clopidogrel plus aspirin versus oral anticoagulation for atrial fibrillation in the Atrial fibrillation Clopidogrel Trial with Irbesartan for prevention of Vascular Events (ACTIVE W): a randomised controlled trial. Lancet 2006;367:1903-12.

24. Friberg L, Rosenqvist M, Lindgren A, et al. High prevalence of atrial fibrillation among patients with ischemic stroke. Stroke 2014;45:2599-605.

25. Healey JS, Connolly SJ, Gold MR, et al. Subclinical atrial fibrillation and the risk of stroke. N Engl 7 Med 2012;366:120-9.

26. Sanna T, Diener HC, Passman RS, et al. Cryptogenic stroke and underlying atrial fibrillation. N Engl 7 Med 2014;370:2478-86.

27. Brambatti M, Connolly SJ, Gold MR, et al. Temporal relationship between subclinical atrial fibrillation and embolic events. Circulation 2014;129:2094-9.

28. Sposato LA, Riccio PM, Hachinski V. Poststroke atrial fibrillation: cause or consequence? Critical review of current views. Neurology 2014;82:1180-6.

29. Higgins P, Dawson J, MacFarlane PW, et al. Predictive value of newly detected atrial fibrillation paroxysms in patients with acute ischemic stroke, for atrial fibrillation after 90 days. Stroke 2014;45:2134-6.

30. Verma A, Cairns JA, Mitchell LB, et al. 2014 Focused update of the Canadian Cardiovascular Society Guidelines for the management of atrial fibrillation. Can 7 Cardiol 2014;30:1114-30.

31. Dowlatshahi D, Butcher KS, Asdaghi N, et al. Poor prognosis in warfarin-associated intracranial hemorrhage despite anticoagulation reversal. Stroke 2012;43:1812-7.

32. Huhtakangas J, Tetri S, Juvela S, et al. Effect of increased warfarin use on warfarinrelated cerebral hemorrhage: a longitudinal population-based study. Stroke 2011:42:2431-5

33. England TJ, Bath PM, Sare GM, et al. Asymptomatic hemorrhagic transformation of infarction and its relationship with functional outcome and stroke subtype: assessment from the Tinzaparin in Acute Ischaemic Stroke Trial. Stroke 2010;41:2834-9.

34. Gladstone DJ, Bui E, Fang J, et al. Potentially preventable strokes in high-risk patients with atrial fibrillation who are not adequately anticoagulated. Stroke 2009;40:235-40

35. Sardar P, Chatterjee S, Wu WC, et al. New oral anticoagulants are not superior to warfarin in secondary prevention of stroke or transient ischemic attacks, but lower the risk of intracranial bleeding: insights from a meta-analysis and indirect treatment comparisons. PLoS ONE 2013;8:e77694.

36. Olesen JB, Lip GY, Hansen ML, et al. Validation of risk stratification schemes for predicting stroke and thromboembolism in patients with atrial fibrillation: nationwide cohort study. BMF 2011;342:d124.

37. Giugliano RP, Ruff CT, Braunwald E, et al. Edoxaban versus warfarin in patients with atrial fibrillation. N Engl 7 Med 2013;369:2093-104.

38. Patel MR, Mahaffey KW, Garg J, et al. Rivaroxaban versus warfarin in nonvalvular atrial fibrillation. NEngl 7 Med 2011;365:883-91.

Affiliations: Department of Clinical Neurosciences (Yu), University of Calgary, Calgary, Alta.; Surveillance and Assessment Branch (Malo, Svenson), Alberta Health, Edmonton, Alta.; Libin Cardiovascular Institute (Wilton), Cumming School of Medicine, University of Calgary, Calgary, Alta.; Division of Cardiology (Parkash), Queen Elizabeth II Health Sciences Center, Halifax, NS; School of Public Health (Svenson), University of Alberta, Edmonton, Alta.; Departments of Clinical Neurosciences and Community Health Sciences (Hill), University of Calgary, Calgary, Alta.

Contributors: Amy Yu and Michael Hill contributed to the conception, design, and interpretation of data and drafted and revised the article. Lawrence Svenson and Shaun Malo contributed to the acquisition, analysis and interpretation of data, and revised the article critically for important intellectual content. Stephen Wilton and Ratika Parkash contributed to data interpretation and critical revision of the article. All of the authors gave final approval to the version to be published and agree to act as guarantors of the work.

Competing interests: Stephen Wilton has consulted for Boehringer Ingelheim and Arca Biopharma; he has received support from St. Jude Medical. Michael D. Hill consults for Merck and has received grant support for clinical trials from Covidien (Medtronic) and HoffmannLa Roche Canada; he has received grant support from Alberta Innovates Health Solutions, Canadian Institutes of Health Research, Heart \& Stroke Foundation of Canada and the National Institutes of Neurological Disorders and Stroke; he reports personal fees from Merck, nonfinancial support from Hoffmann-La Roche Canada and grants from Covidien (Medtronic), outside the submitted work; he has a patent pending with the US Patent Office (no. 62/086,077) and owns stock in Calgary Scientific Incorporated, a company that focuses on medical imaging software. No other competing interests were declared.

Supplemental information: For reviewer comments and the original submission of this manuscript, please see www.cmajopen.ca/content/4/1/ E1/suppl/DC1 